\title{
Article
}

\section{Quantities of GLP-1 mimetics}

Davies, Janice Anne

Available at http://clok.uclan.ac.uk/32634/

Davies, Janice Anne (2020) Quantities of GLP-1 mimetics. Journal of Prescribing Practice, 2 (3). p. 120. ISSN 2631-8385

It is advisable to refer to the publisher's version if you intend to cite from the work.

10.12968/jprp.2020.2.3.120

For more information about UCLan's research in this area go to

http://www.uclan.ac.uk/researchgroups/ and search for < name of research Group>.

For information about Research generally at UCLan please go to http://www.uclan.ac.uk/research/

All outputs in CLoK are protected by Intellectual Property Rights law, including Copyright law. Copyright, IPR and Moral Rights for the works on this site are retained by the individual authors and/or other copyright owners. Terms and conditions for use of this material are defined in the policies page.

\section{CLoK}

Central Lancashire online Knowledge www.clok.uclan.ac.uk

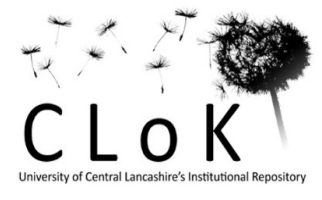


It is important to ensure that appropriate quantities of GLP1 mimetics are prescribed to avoid financial waste and for patient safety reasons. Six GLP1 mimetics are licensed for diabetes management. (PrescQIPP, 2019)

Calculate the number of devices needed for a one month supply for each GLP1 mimetic.

\begin{tabular}{|c|c|c|c|c|}
\hline $\begin{array}{l}\text { Generic name (and } \\
\text { brand name) of GLP1 } \\
\text { mimetic }\end{array}$ & Dose & $\begin{array}{l}\text { Strength and volume } \\
\text { of device }\end{array}$ & \multicolumn{2}{|c|}{$\begin{array}{l}\text { How many devices } \\
\text { are needed for one } \\
\text { month? }\end{array}$} \\
\hline \multirow[t]{2}{*}{ Exenatide (Byetta) } & \multirow{2}{*}{$\begin{array}{l}5 \text { micrograms twice } \\
\text { daily before meals, } \\
\text { increased if } \\
\text { necessary to } 10 \\
\text { micrograms twice } \\
\text { daily }\end{array}$} & \multirow{2}{*}{$\begin{array}{l}5 \mathrm{micrograms} / 0.02 \mathrm{ml} \text {, } \\
1.2 \mathrm{ml} \text { pre-filled pen } \\
10 \mathrm{micrograms} / 0.02 \mathrm{ml} \text {, } \\
2.4 \mathrm{ml} \text { pre-filled pen }\end{array}$} & $\begin{array}{l}5 \\
\text { microgram } \\
\text { dose }\end{array}$ & $\begin{array}{l}\text { Answer = } \\
1 \text { pre- } \\
\text { filled pen }\end{array}$ \\
\hline & & & $\begin{array}{l}10 \\
\text { microgram } \\
\text { dose }\end{array}$ & $\begin{array}{l}\text { Answer = } \\
1 \text { pre- } \\
\text { filled pen }\end{array}$ \\
\hline Exenatide (Byduron) & $2 \mathrm{mg}$ once weekly & $\begin{array}{l}\text { Pre-filled injection } \\
\text { injection containing } \\
2 \mathrm{mg} \text { powder and } \\
\text { solvent for prolonged } \\
\text { release injection }\end{array}$ & \multicolumn{2}{|c|}{$\begin{array}{l}\text { Answer }=4 \text { pre-filled } \\
\text { disposable injections }\end{array}$} \\
\hline \multirow[t]{2}{*}{ Liraglutide (Victoza) } & \multirow{2}{*}{$\begin{array}{l}\text { Initially } 0.6 \mathrm{mg} \text { once a } \\
\text { day for at least a } \\
\text { week, then increased } \\
\text { to } 1.2 \mathrm{mg} \text { daily for a } \\
\text { week, increased if } \\
\text { necessary to } 1.8 \mathrm{mg} \\
\text { daily }\end{array}$} & \multirow[t]{2}{*}{$\begin{array}{l}6 \mathrm{mg} / \mathrm{ml} 3 \mathrm{ml} \text { prefilled } \\
\text { pen }\end{array}$} & $\begin{array}{l}1.2 \mathrm{mg} \\
\text { dose }\end{array}$ & $\begin{array}{l}\text { Answer = } \\
2 \text { pre- } \\
\text { filled } \\
\text { pens }\end{array}$ \\
\hline & & & $\begin{array}{l}1.8 \mathrm{mg} \\
\text { dose }\end{array}$ & $\begin{array}{l}\text { Answer = } \\
3 \text { pre- } \\
\text { filled } \\
\text { pens }\end{array}$ \\
\hline \multirow[t]{2}{*}{ Lixisenatide Lyxumia) } & \multirow[t]{2}{*}{$\begin{array}{l}\text { Initially } 10 \\
\text { micrograms once } \\
\text { daily for } 2 \text { weeks, } \\
\text { then } 20 \text { micrograms } \\
\text { once daily }\end{array}$} & $\begin{array}{l}10 \text { microgram } / 0.2 \mathrm{ml} \\
3 \mathrm{ml} \text { pre-filled pen } \\
\text { (titration pack) }\end{array}$ & $\begin{array}{l}10 \\
\text { microgram } \\
\text { titration } \\
\text { dose }\end{array}$ & $\begin{array}{l}\text { Answer = } \\
1 \text { pre- } \\
\text { filled pen }\end{array}$ \\
\hline & & $\begin{array}{l}20 \text { microgram } / 0.2 \mathrm{ml} 3 \\
\mathrm{ml} \text { pre-filled pen }\end{array}$ & $\begin{array}{l}20 \\
\text { microgram } \\
\text { dose }\end{array}$ & $\begin{array}{l}\text { Answer = } \\
2 \text { pre- } \\
\text { filled } \\
\text { pens }\end{array}$ \\
\hline \multirow[t]{2}{*}{ Dulaglutide (Trulicity) } & \multirow[t]{2}{*}{$\begin{array}{l}0.75 \mathrm{mg} \text { once weekly } \\
\text { as monotherapy } \\
1.5 \mathrm{mg} \text { once weekly in } \\
\text { combination }\end{array}$} & \multirow{2}{*}{$\begin{array}{l}0.75 \mathrm{mg} / 0.5 \mathrm{ml} \text { pre- } \\
\text { filled pen } \\
\begin{array}{l}1.5 \mathrm{mg} / 0.5 \mathrm{ml} \text { pre-filled } \\
\text { pen }\end{array}\end{array}$} & $\begin{array}{l}0.75 \mathrm{mg} \\
\text { dose }\end{array}$ & $\begin{array}{l}\text { Answer = } \\
4 \text { pre- } \\
\text { filled } \\
\text { pens }\end{array}$ \\
\hline & & & $\begin{array}{l}1.5 \mathrm{mg} \\
\text { dose }\end{array}$ & $\begin{array}{l}\text { Answer }= \\
4 \text { pre- }\end{array}$ \\
\hline
\end{tabular}




\begin{tabular}{|l|l|l|l|l|}
\hline & & & & $\begin{array}{l}\text { filled } \\
\text { pens }\end{array}$ \\
\hline $\begin{array}{l}\text { Semaglutide } \\
\text { (ozempic) }\end{array}$ & $\begin{array}{l}\text { Initially } 0.25 \mathrm{mg} \text { once } \\
\text { weekly for 4 weeks, } \\
\text { then } 0.5 \mathrm{mg} \text { once } \\
\text { weekly for at least } 4 \\
\text { weeks, then if } \\
\text { necessary } 1 \mathrm{mg} \text { once } \\
\text { weekly }\end{array}$ & $\begin{array}{l}0.5 \mathrm{mg} / 0.37 \mathrm{ml} 1.5 \mathrm{ml} \\
\text { pre-filled pen } \\
\text { filled pen }\end{array}$ & $\begin{array}{l}0.5 \mathrm{mg} \\
\text { dose }\end{array}$ & $\begin{array}{l}\text { Answer }= \\
1 \text { pre- } \\
\text { filled pen }\end{array}$ \\
\cline { 3 - 5 } & & $\begin{array}{l}\text { Answer }= \\
1 \text { pre- } \\
\text { filled pen }\end{array}$ \\
\hline
\end{tabular}

Ensuring appropriate quantities of GLP1 mimetics, 2019, PrescQIPP

https://www.prescqipp.info/our-resources/webkits/hot-topics/ 